\title{
Lattice QCD at finite temperature
}

\author{
Shinji Ejiri ${ }^{a}$

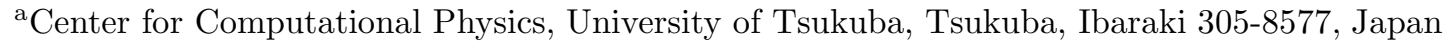

Recent developments in finite-temperature QCD with dynamical quarks are reviewed focusing on the topics of critical temperature, the equation of state, and critical behaviors around the chiral phase transition.

\section{Introduction}

In the last years, much effort has been paid to experimentally detect the quark-gluon plasma (QGP) in heavy-ion collisions. In these experiments, precise theoretical inputs about the nature of the transition and the equation of state (EOS) of QGP are indispensable to unambiguously identify the signal of QGP. Here, lattice QCD provides us with the most powerful method to compute thermodynamic properties of QGP from the first principles of QCD.

In the quenched approximation of $\mathrm{QCD}$, the study of finite temperature QCD is already well matured: Computations of $T_{c}$ and EOS have been made on lattices with several temporal lattice sizes $N_{t}$, using both the standard plaquette action and various improved actions. Continuum extrapolation to the limit of large $N_{t}$ has been performed with different actions to confirm good agreement within an accuracy of a few percent 1-3. For the pressure, precise comparisons of the results from different calculation methods, i.e. the integral method泊 and the derivative method [5], have also been made [6] 8]. The expected absence of the pressure gap at the first order deconfinement transition point has also been demonstrated through a new non-perturbative calculation of anisotropy coefficients|[7].

The quenched study is now entering a new stage: Thermal effects on the pole mass have been studied by the QCDTARO collaboration using an anisotropic lattice 9]. Several groups are also trying to compute hadron spectral functions at finite-temperature applying the maximum entropy method 10 13.

On the other hand, the study of full QCD at finite temperatures is still at the stage of development. Until recently, EOS with dynamical quarks has been computed only for the staggered fermion. The MILC collaboration calculated EOS in 2 flavor QCD with the standard staggered fermion on $N_{t}=4$ and 6 lattices 14]. The Bielefeld group computed EOS for $N_{f}=4$ using an improved staggered fermion at $N_{t}=4[15]$. This year, the Bielefeld group extended the computation to $N_{f}=2$ and 3 and studied the $N_{f^{-}}$ dependence 16.

A new development of this year is the first calculation of EOS for a Wilson-type fermion 17. Using a renormalization group (RG) improved gauge action 18] coupled with 2 flavors of cloverimproved Wilson quarks 19], the CP-PACS collaboration performed a systematic study of the pressure and the energy density of QGP.

In this report, we concentrate on the development in finite temperature QCD with dynamical quarks, focusing on the topics of EOS, and attempt a comparison of the results from different lattice fermions. In section 2, we discuss about the phase structure, critical properties around the chiral phase transition of 2 flavor QCD, and the transition temperature. we then discuss new calculations of EOS in section 3. A brief summary is given in section 4 .

\section{Phase structure}

\subsection{Phase structure with Wilson fermions}

It has been difficult to study EOS with Wilsontype quarks due to several difficulties: One is the complicated phase structure due to the explicit violation of chiral symmetry and due to the appearance of the parity-flavor broken phase (Aoki 


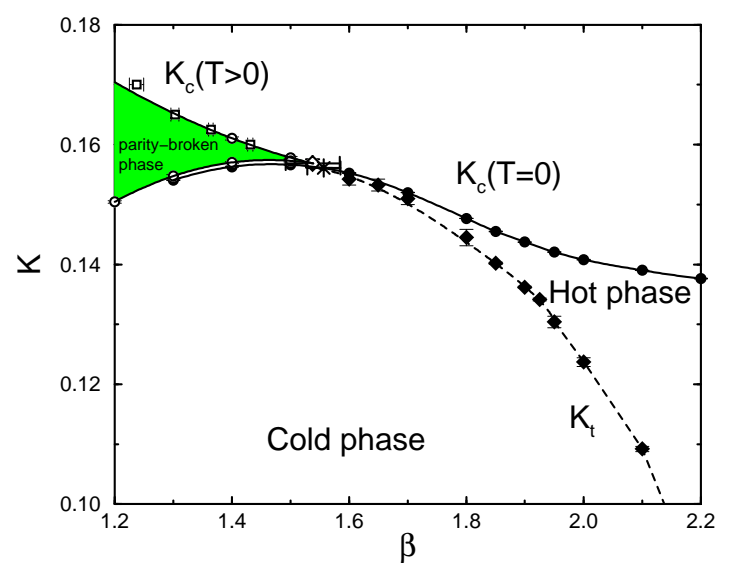

Figure 1. Phase diagram for the RG-improved gauge action and the clover-improved Wilson quark action on an $N_{t}=4$ lattice 24.

phase) 20,21]. Therefore, a systematic study surveying a wide range of the parameter space is required. The second reason is that, when the standard plaquette gauge action and the standard Wilson quark action are used, lattice artifacts are large on coarse lattices used in most finite temperature simulations, For example, unexpected strong phase transition is observed at intermediate quark masses in 2 flavor QCD 22, 23]. Therefore, we have to use improved actions to suppress these lattice artifacts.

The CP-PACS collaboration studied 2 flavor QCD with an RG-improved gauge action combined with a clover-improved Wilson quark action 24. The first step is to clarify the phase structure for this combination of actions.

Figure 1 is the result for the phase diagram of QCD obtained on an $N_{t}=4$ lattice. The solid line $K_{c}(T=0)$ is the location of the chilal limit at zero temperature. The pion mass decreases as $K$ is increased from small $K$, and vanishes on the line $K_{c}(T=0)$. When $N_{t}$ is fixed as in most simulations, the temperature $T=\left(N_{t} a\right)^{-1}$ becomes higher as $\beta$ is increased. The dashed line $K_{t}$ is the pseudo-critical line for the finite temperature phase transition for $N_{t}=4$. The region to the right of $K_{t}$ (larger $\beta$ ) is the high temperature QGP phase, and that to the left of $K_{t}$ (smaller $\beta$ ) is the low temperature hadron phase. The cross-

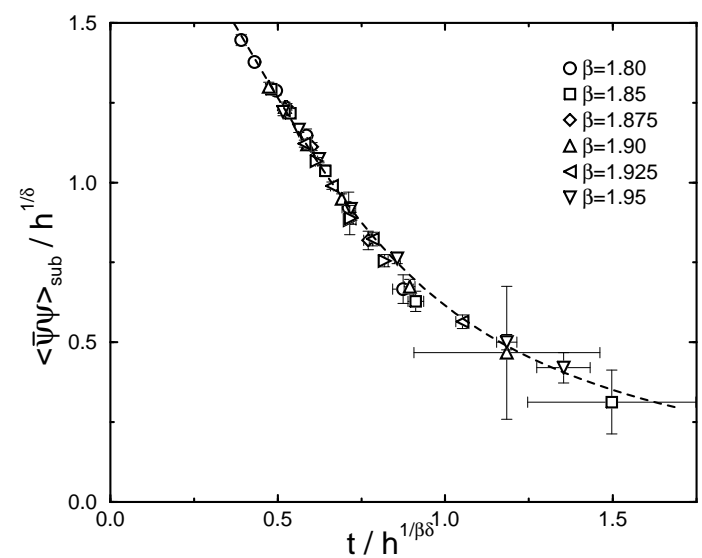

Figure 2. $\quad \mathrm{O}(4)$ scaling relation measured on a $16 \times 4$ lattice with the RG gauge and clover quark actions 24.

ing point of the $K_{c}(T=0)$ and the $K_{t}$ lines is the chiral phase transition point 23].

In the region above the $K_{c}(T=0)$ line, a parity-flavor symmetry of the Wilson-type quark action is broken spontaneously 20,21. At zero temperature, the boundary of the parity-flavor broken phase (the $K_{c}(T=0)$ line) is known to form a sharp cusp touching the free massless fermion point $K=1 / 8$ at $\beta=\infty$.

At finite temperatures, the parity-flavor broken phase retracts from the large $\beta$ region. The boundary of the parity-flavor broken phase at $T>0$ (the $K_{c}(T>0)$ line) is shown in Fig. 1 for $N_{t}=4$. As shown in this figure, the chiral transition point locates close to the cusp of the parity-flavor broken phase, the difference being at most of $O(a)$. This is consistent with the picture that the massless pion, i.e. the Goldstone boson associated with spontaneous chiral symmetry breaking, appears only in the cold phase.

\subsection{Scaling study of 2 flavor QCD}

An important step towards clarification of the QCD transition in the real world is the study of the finite temperature chiral phase transition in $N_{f}=2$ QCD. When the chiral transition in $N_{f}=$ 2 QCD is second order, the transition is expected to be universal to that in a 3-dimensional $\mathrm{O}(4)$ spin model: With the identifications $M \sim\langle\bar{\Psi} \Psi\rangle$ 
for the magnetization, $h \sim m_{q} a$ for the external magnetic field and $t \sim \beta-\beta_{c t}$ for the reduced temperature, where $\beta_{c t}$ is the chiral transition point, we expect the same scaling behavior as the $\mathrm{O}(4)$ spin model. Therefore, confirmation of the $\mathrm{O}(4)$ scaling in QCD provides us with a test of the order of the chiral transition.

The $\mathrm{O}(4)$ scaling was first tested with staggered fermion by Karsch and Laermann[25]. The study was extended to a wider range of the quark mass and lattice sizes by the JLQCD collaboration [26] and by the Bielefeld group [27]. However, an agreement of the critical exponents between the $\mathrm{O}(4)$ spin model and QCD with 2 flavors of staggered fermions was not obtained for $N_{t}=4$.

For the case of Wilson fermion, Iwasaki et al.228] investigated the scaling relation;

$M / h^{1 / \delta}=f\left(t / h^{1 / \beta \delta}\right)$

using the RG gauge action combined with the standard Wilson quark action. They identified the subtracted chiral condensate defined by an axial Ward-Takahashi identity 29]

$\langle\bar{\Psi} \Psi\rangle_{\mathrm{sub}}=2 m_{q} a Z \sum_{x}\langle\pi(x) \pi(0)\rangle$,

as the magnetization of the spin model. Here, the quark mass $m_{q}$ is defined by an axial vector Ward-Takahashi identity 29.30], and the treelevel renormalization coefficient $Z=(2 K)^{2}$ was adopted. They found that the scaling relation (1) is well satisfied with the $\mathrm{O}(4)$ critical exponents and the $\mathrm{O}(4)$ scaling function.

Figure 2 is the new result for the case of the clover-improved Wilson quark action coupled with the RG gauge action, obtained on a $16^{3} \times 4$ lattice 24. The vertical axis is $M / h^{1 / \delta}$ and the horizontal axis is $t / h^{1 / \beta \delta}$, where $\beta$ and $\delta$ are the critical exponent obtained in the $\mathrm{O}(4)$ spin model[31]. The dashed line is the $\mathrm{O}(4)$ scaling function [32]. They fitted the data to the scaling function adjusting $\beta_{c t}$ and the scales of two axes. As seen from Fig. 2, QCD data is well described by the $\mathrm{O}(4)$ scaling ansatz also for this combination of actions. The best fit gives $\beta_{c t}=1.469$ (73) for the chiral transition point, with $\chi^{2} / N_{D F}=0.82$. This result suggests that the chiral transition is of second order for $N_{f}=2$.

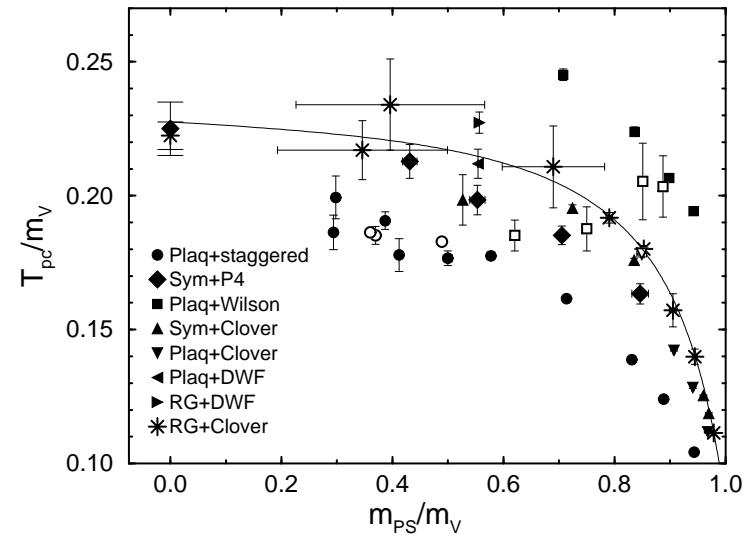

Figure 3. $\quad T_{p c} / m_{V}$ as a function of $m_{P S} / m_{V}$ with various actions.

This year, the MILC collaboration published their final results of a study of scaling properties in QCD with standard staggered quarks 33. They found that the QCD data are inconsistent with the $\mathrm{O}(4)$ scaling, in accord with the previous results for the critical exponents 27,26. At the same time, a comparison of the results for $N_{t}=4-12$ suggests that the discrepancy with the $\mathrm{O}(4)$ scaling function may be removed when $N_{t}$ is sufficiently large.

A different approach to clarify the origin of the unexpected results was proposed by Kogut, Lagaë and Sinclair 34. They carried out a simulation at precisely zero quark mass by adding the additional, irrelevant four-fermi interaction to QCD. From their results obtained on $N_{t}=4$ and 6 lattices, they also suggested importance of simulations at larger $N_{t}$.

For staggered quarks, a comparison with an $\mathrm{O}(2)$ spin model is also important because the symmetry of the staggered quark action is $\mathrm{O}(2)$ at finite lattice spacings. Engels et al. calculated the scaling function for the $\mathrm{O}(2)$ spin model[35]. They found that the $\mathrm{O}(2)$ scaling function is quite similar to the $\mathrm{O}(4)$ scaling function. This implies that the $\mathrm{O}(2)$ scaling also cannot explain the behavior of the staggered quarks at small $N_{t}$.

\subsection{Transition temperature}

In Fig. 3, we summarize the results of the pseudo-critical transition temperature $T_{p c}$ in 
Table 1

Chiral transition temperature $T_{c}$ in physical units. The scale is fixed by $\sqrt{\sigma} \simeq 425 \mathrm{MeV}$ for quenched $\left(N_{f}=0\right)$ QCD, and by $m_{V}=770 \mathrm{MeV}$ for $N_{f}=2$ and 3 QCD.

\begin{tabular}{ccccccc}
\hline action & Ref. & $N_{f}$ & $N_{t}$ & $T_{c} / \sqrt{\sigma}$ & $T_{c} / m_{V}$ & $T_{c}(\mathrm{MeV})$ \\
\hline plaquette & 2,41 & 0 & $\infty$ & $0.630(5)$ & & 268 \\
Symanzik & 2 & 0 & $\infty$ & $0.634(4)$ & & 269 \\
RG & 3 & 0 & $\infty$ & $0.650(5)$ & & 276 \\
RG+clover & 24 & 2 & 4 & & $0.222(5)$ & $171(4)$ \\
Symanzik+p4 & 38 & 2 & 4 & $0.425(15)$ & $0.225(10)$ & $173(8)$ \\
Symanzik+p4 & {$[38$} & 3 & 4 & & $0.20(1)$ & $154(8)$ \\
\hline
\end{tabular}

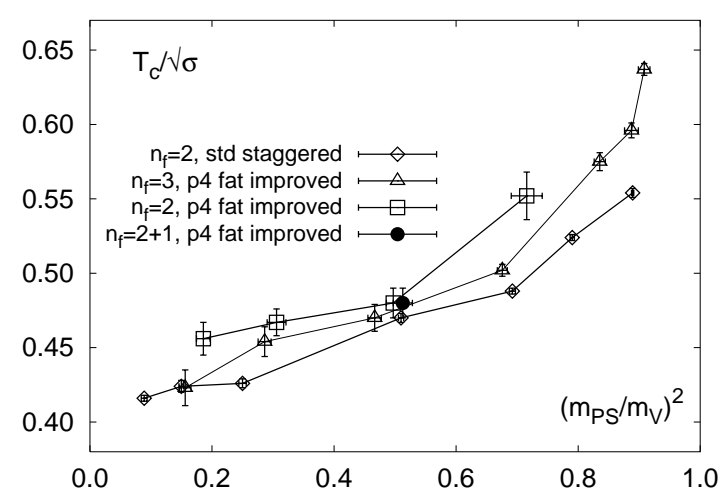

Figure 4. Pseudo-critical temperature $T_{p c}$ in a unit of $\sqrt{\sigma}$ for $2,3,2+1$ flavor QCD using the p4-improved staggered action 38.

$N_{f}=2$ QCD with various actions; plaquette gauge combined with the standard staggered and Wilson quark 36, 37], Symanzik gauge with p4-improved staggered quark 16, 38, plaquette gauge with clover quark [39], Symanik gauge with clover quark [37], RG gauge with clover quark [24], and plaquette and RG gauge with domain-wall quark 40]. The scale is fixed by the vector meson mass $m_{V}$ at the simulation point. Filled and open symbols are respectively the results on $N_{t}=4$ and 6 lattices. Diamonds and stars are new; the diamonds from the p4-improved staggered quark by the Bielefeld group 38, and the stars from the RG gauge with clover quark by the CP-PACS collaboration 24. We note that the new results are slightly larger than the previous results from the standard staggered quarks presented by circles. The Bielefeld group and the CP-PACS collabo- ration also attempted chiral extrapolations. The results agree with each other within errors in the chiral limit, giving $T_{c} \sim 170 \mathrm{MeV}$ for the chiral transition temperature.

The Bielefeld group also investigated the $N_{f^{-}}$ dependence of $T_{p c}$ on a $16^{3} \times 4$ lattice 38. Figure 6 shows their results for 2 (square) and 3 (triangle) flavors of degenerate staggered quarks, normalized by the string tension. From this figure, the dependence on $N_{f}$ seems to be small for $N_{f}=2-3$.

Table 1 is a summary of the transition temperature in the chiral limit. The quenched results are extrapolated to the continuum limit. However, those for full QCD, $T_{c} \sim 150-180 \mathrm{MeV}$, are the results for $N_{t}=4$, and confirmation at lager $N_{t}$ is required.

\section{Equation of state}

The equation of state (EOS) is one of the most basic information in a phenomenological study of QGP. In this section, we discuss the latest results for EOS from full QCD. Among various methods to compute the pressure, the integral method [4] is commonly used in recent investigations. This method is based on the equation, $p=-f$, valid for large homogeneous systems, where $f=(-T / V) \ln Z$ is the free energy density. Because the derivatives of the partition function can be expressed by expectation values of operators, which are computable by a Monte-Carlo simulation, we obtain the pressure by integrating this expectation value in the parameter space. 

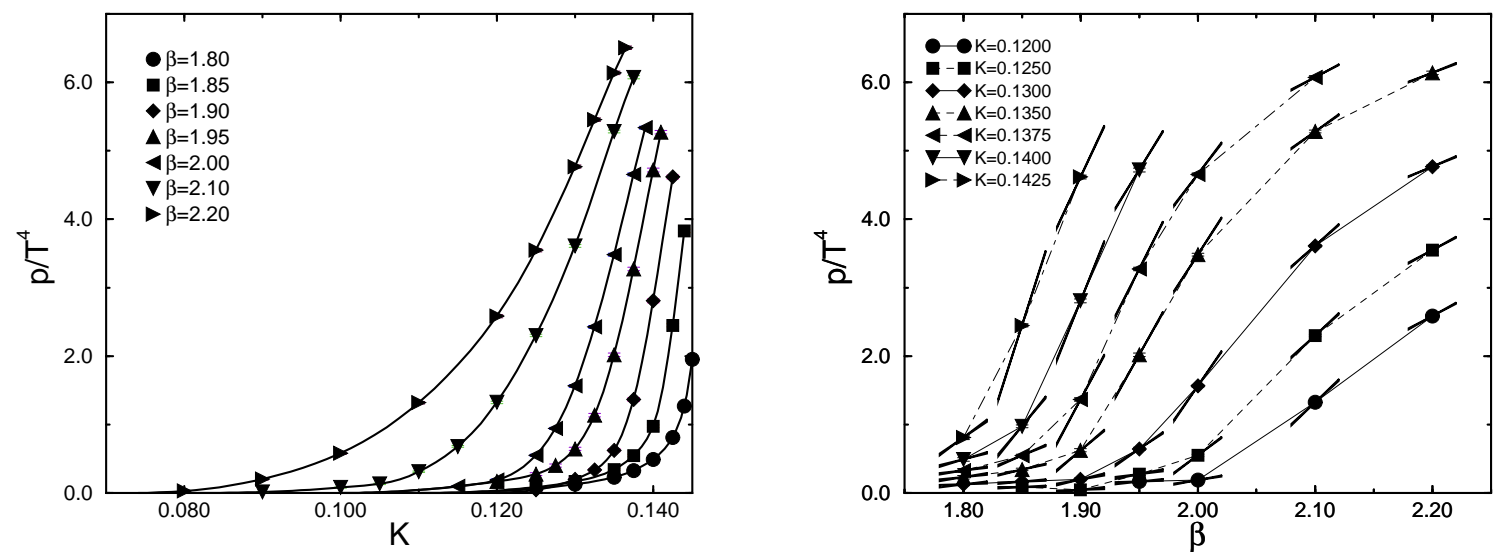

Figure 5. Pressure calculated with RG gauge and clover quark actions on a $16^{3} \times 4$ lattice, as a function of $K$ (left) and $\beta$ (right). The short lines on the symbols in the right figure express the slope of the pressure in the $\beta$-direction, calculated independently 17 .

For the case of the Wilson quark, we have

$$
\begin{gathered}
\frac{p}{T^{4}}=-\frac{f}{T^{4}}=-N_{t}^{4} \int^{(\beta, K)} \mathrm{d} \xi\left\{\frac{1}{N_{s}^{3} N_{t}}\left\langle\frac{\partial S}{\partial \xi}\right\rangle\right. \\
-(\text { value at } T=0)\}
\end{gathered}
$$

with $\mathrm{d} \xi=\left(\mathrm{d} \beta^{\prime}, \mathrm{d} K^{\prime}\right)$ on the integration path. The starting point of the integration path should be chosen such that $p \approx 0$ there.

\subsection{Equation of state with Wilson quarks}

The CP-PACS collaboration carried out a systematic calculation of EOS in $N_{f}=2$ full QCD, using the RG gauge and the clover quark actions on $16^{3} \times 4$ and $16^{3} \times 6$ lattices 17. Simulations at $T=0$ for subtraction and also to fix the scale and the line of constant physics, were made on a $16^{4}$ lattice. The derivatives of the action in $\beta$ and $K$ were computed using a U(1) noise method. They found that the derivative in the $K$-direction gives a smaller error in the final pressure. Therefore, they chose the integral path in the $K$-direction. Figure 5 (left) is the $K$-dependence of $p / T^{4}$ for various $\beta$. In order to check the reliability of this method, they also show the data as a function of $\beta$ in Fig. 目(right). The short lines on the symbols are the slope of the pressure in terms of $\beta$, which are measured directly from $\langle\partial S / \partial \beta\rangle$. We see that the slopes well describe the pressure curves which are computed from an independent measurement of $\langle\partial S / \partial K\rangle$. This means that the value of the

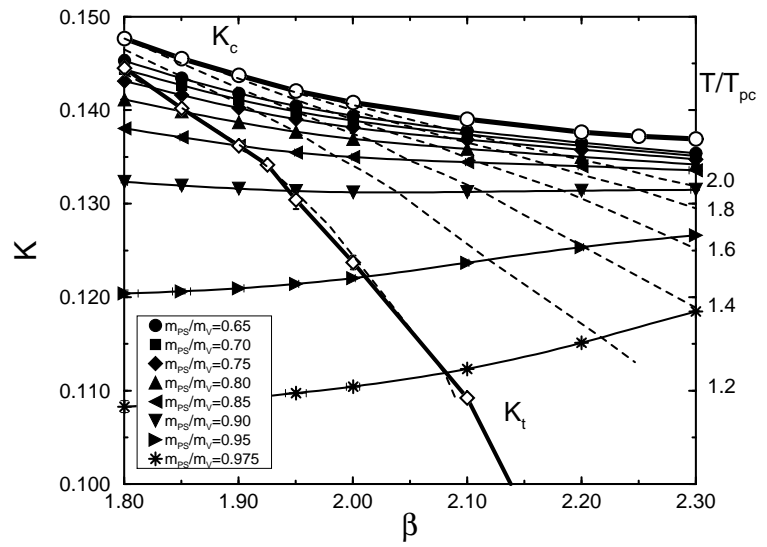

Figure 6. $\quad m_{P S} / m_{V}$ constant lines and $T / T_{p c}$ constant lines for an $N_{t}=4$ lattice in the $(\beta, K)$ parameter space. The values of $T / T_{p c}$ for the dashed lines are given on the right edge of the figure 17.

pressure is independent of the choice of the integration path in the parameter space.

The data in this form is not yet useful for phenomenological applications. We wish to calculate the temperature dependence for each physical system, i.e., on a line of constant physics. The CP-PACS collaboration has chosen the condition $m_{P S} / m_{V}=$ constant, determined at $T=0$, to define the lines of constant physics. The relation between the simulation parameters and the phys- 


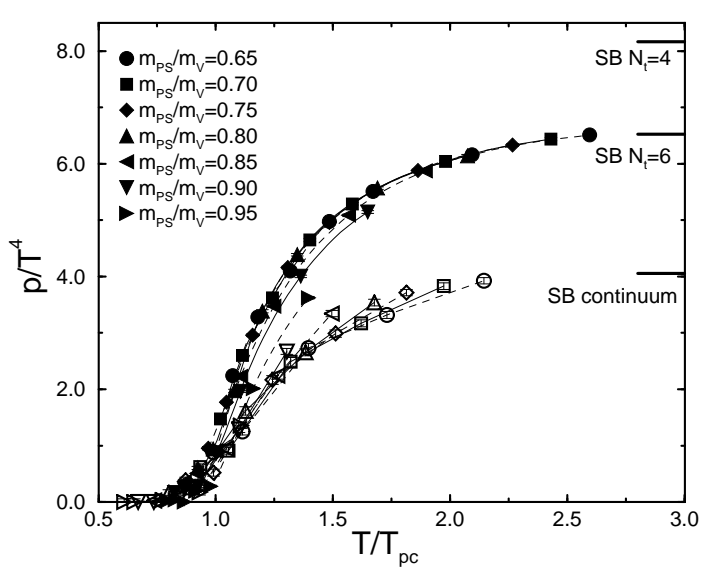

Figure 7. Pressure calculated with the RG gauge and clover quark actions on $16^{3} \times 4$ and $16^{3} \times 6$ lattices, as a function of $T / T_{p c} \llbracket 17$.

ical parameters is then given by Fig. 6. The solid lines are the lines of constant physics. The dashed lines are $T / T_{p c}=$ constant lines, where $T_{p c}$ is the pseudo-critical temperature on the same line of constant physics and the scale was fixed by $m_{V}$. The dashed line $T / T_{p c}=1$ itself was determined by fitting the pseudo-critical transition points (diamonds) obtained on an $N_{t}=4$ lattice.

Interpolating the data in Fig. 5 yields Fig. 0 in which the pressure is given as a function of temperature, for each line of constant physics. Filled (open) symbols are the results of $N_{t}=4$ (6). Different shapes of the symbol correspond to different values of $m_{P S} / m_{V}$, i.e., different quark masses. This figure shows that the pressure is almost independent of the quark mass in a wide range of $m_{P S} / m_{V}$. On the other hand, the $N_{t^{-}}$ dependence is sizeable: there exist large lattice artifacts in the $N_{t}=4$ results.

The energy density is obtained by combining the results for the pressure and the results for the quantity;

$$
\begin{aligned}
\frac{\epsilon-3 p}{T^{4}}=N_{t}^{4}\left[\frac{1}{N_{s}^{3} N_{t}}\left(a \frac{\partial \beta}{\partial a}\left\langle\frac{\partial S}{\partial \beta}\right\rangle+a \frac{\partial K}{\partial a}\left\langle\frac{\partial S}{\partial K}\right\rangle\right)\right. \\
-(\text { value at } T=0)] .
\end{aligned}
$$

Here, the beta functions, $a \frac{\partial \beta}{\partial a}$ and $a \frac{\partial K}{\partial a}$, can be determined by measuring the differentials of $\beta$ and $K$ in terms of the $m_{V} a$, on each $m_{P S} / m_{V}=$

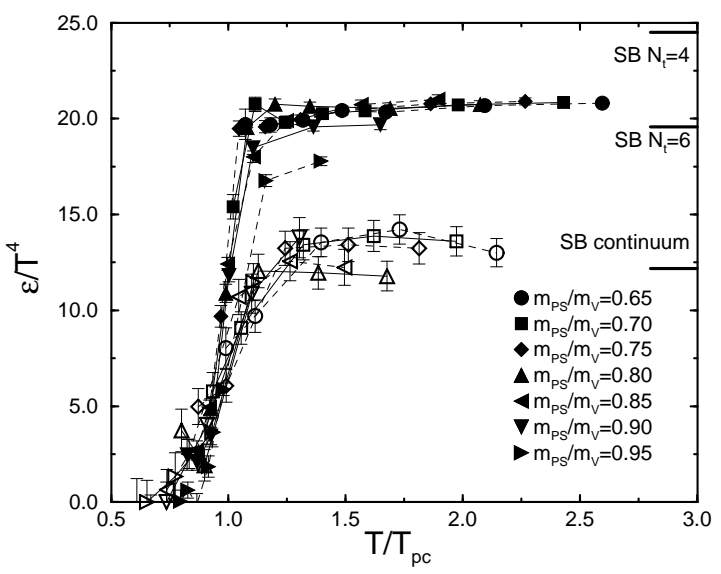

Figure 8. Energy density as a function of $T / T_{p c}$, calculated with RG gauge and clover quark actions on $16^{3} \times 4$ and $16^{3} \times 6$ lattices [17].

constant line.

Figure 8 is the final results for the energy density as a function of the temperature. They indicate that the quark mass dependence for the energy density is also small.

Previous results from the staggered quark showed a peak just above $T_{c}$ on an $N_{t}=4$ lattice 14. We do not see such a peak in Fig. 8 from the improved Wilson quark. Also the peak disappears at $N_{t}=6$ for the staggered fermion, thus, the peak seems to be a lattice artifact.

We find that the magnitude and $T$-dependence of EOS on $N_{t}=6$ lattices are quite similar between improved Wilson and staggered quarks. The quark mass dependence was also investigated with the staggered quark; similar to the case of the improved Wilson quark, the difference between $m_{q} / T=0.075$ and 0.15 is found to be small within errors 14].

Short lines on the right axes of Figs. 7 and 8 are the Stefan-Boltzmann (SB) values at $N_{t}=4$, 6 and in the continuum limit. Both $N_{t}=4$ and 6 results do not indicate a clear approach towards these values in the high temperature limit. A clear deviation from the SB limits at finite $N_{t}$ was also observed in a quenched study of EOS with the RG-improved action[3]. Further study is necessary to clarify this phenomenon. However, quenched studies show that EOS extrapolated to 


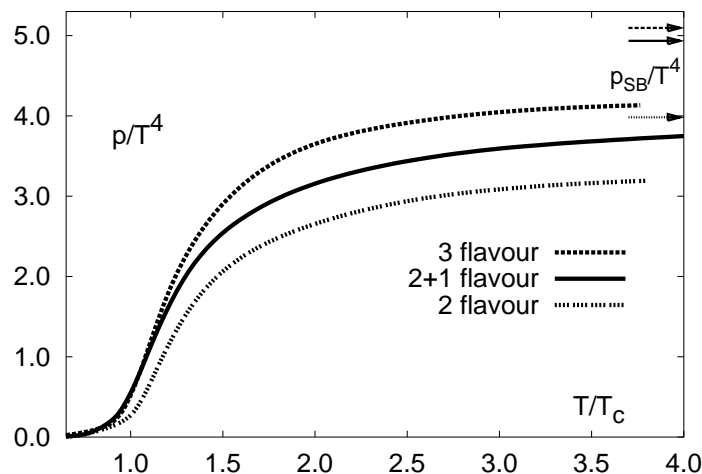

Figure 9. Pressure from the p4-improved staggered action on a $16^{3} \times 4$ lattice as a function of $T / T_{p c}$ 16].

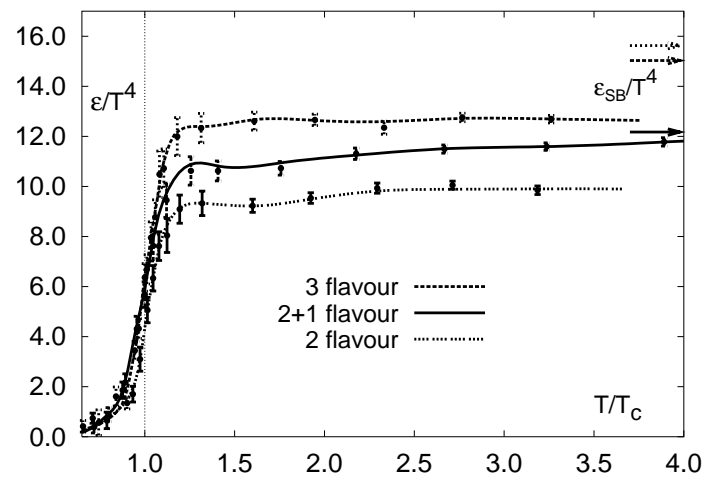

Figure 10. Energy density from the p4-improved staggered action on a $16^{3} \times 4$ lattice as a function of $T / T_{p c}$ 16.

the continuum limit $N_{t}=\infty$ is consistent with the continuum SB value in the high temperature limit. From Figs. 7 and 8 , we note that the results for $N_{t}=6$ are already close to the continuum SB limit. An accurate continuum extrapolation may be possible already with data at $N_{t} \sim 8$ or 10 .

\section{2. $N_{f}$-dependence of equation of state}

So far, we have discussed simulations with 2 dynamical quarks, which are identified as light up and down quarks. In this subsection, We review results on the effect of the strange quark. The Bielefeld group compared EOS for $N_{f}=2,3$ and $2+1$ on a $16^{3} \times 4$ lattice, using the p4-improved staggered quark action with the Symanzik improved gauge action 16]. $\quad N_{t^{-}}$ dependence is known to be small for this action in the Stefan-Boltzmann limit. They computed EOS at $m_{q} / T=0.4$ for $N_{f}=2$ and 3 , and at $m_{u, d} / T=0.4$ and $m_{s} / T=1.0$ for $N_{f}=2+1$.

Figure 9 shows the results of pressure. A clear $N_{f}$-dependence is visible; the pressure becomes larger as $N_{f}$ is increased.

Clear $N_{f}$-dependence was observed also in the energy density. They also observed peaks of the energy density near $T_{p c}$, similar to the result of the MILC collaboration at $N_{t}=4$.

They attempted to estimate the energy density in the chiral limit by subtracting the contribution of $\left\langle\partial S / \partial\left(m_{q} a\right)\right\rangle$ in the calculation of the $(\epsilon-3 p) / T^{4}$, since this term vanishes when the quark mass is zero. Quark mass dependence was assumed to be small in the pressure. Figure 10 shows the resulting estimate of $\epsilon / T^{4}$ in the chiral limit. The peak disappeared in this figure. Therefore, in contrast to the case of Fig. 息, noticeable quark mass dependence was suggested for the energy density. Further study is necessary to clarify this point.

\section{Summary}

We reviewed progress of the numerical study of finite-temperature QCD with dynamical quarks in the past year, focusing on the topics of EOS.

For the transition temperature, recent results for $N_{f}=2 \mathrm{QCD}$, computed with improved staggered and improved Wilson fermion actions on $N_{t}=4$ lattices, agree well with each other. In the chiral limit, the transition temperature is about $170 \mathrm{MeV}$. This value is higher than the previous results from the standard staggered fermion at $N_{t}=4$ and 6 . Confirmation of this result requires further study at lager $N_{t}$.

The first calculation of EOS with Wilson-type fermion was performed for $N_{f}=2$ at $N_{t}=4$ and 6 , using an improved Wilson fermion. The results of EOS for $N_{t}=6$ are quite similar between the improved Wilson and the staggered fermions, and are close to the continuum Stephan-Boltzmann value at high temperatures. This may suggest that a small $N_{t} \sim 8$ or 10 is already sufficient for a precise calculation of EOS. On the other 
hand, a clear $N_{f}$-dependence was reported for EOS. This implies the importance of including dynamical strange quark in the simulations.

In summary, we have obtained two keywords for the next step of full QCD thermodynamics: larger $N_{t}$ with improved actions towards the continuum limit, and simulations with the dynamical strange quark. Studies in these directions are now starting.

I would like to thank A. Ukawa and K. Kanaya for helpful suggestion and comments on the manuscript. I also thank F. Karsch for communication on their results and useful discussions. This work is supported by JSPS.

\section{REFERENCES}

1. G. Boyd et al., Phys. Rev. Let. 75 (1995) 4169; Nucl. Phys. B469 (1996) 419.

2. B. Beinlich et al., Eur. Phys. J. C6 (1999) 133.

3. CP-PACS Collaboration: M. Okamoto et al., Phys. Rev. D60 (1999) 094510.

4. J. Engels et al., Phys. Lett. B252 (1990) 625.

5. J. Engels et al., Nucl. Phys. B205 (1982) 545.

6. T.R. Klassen, Nucl. Phys. B533 (1998) 557.

7. S. Ejiri, Y. Iwasaki, and K. Kanaya, Phys. Rev. D58 (1998) 094505.

8. J. Engels, F. Karsch, and T. Scheideler, Nucl. Phys. B564 (2000) 303.

9. QCDTARO Collaboration: Ph. de Forcrand et al., hep-lat/9901017; hep-lat/000805; T. Umeda et al., these proceedings, heplat/0010090.

10. QCDTARO Collaboration: Ph.de Forcrand et al., Nucl. Phys. B(Proc.Suppl.) 63 (1998) 460.

11. Y. Nakahara, M. Asakawa, and T. Hatsuda, Phys. Rev. D60 (1999) 091503.

12. I. Wetzorke and F. Karsch, hep-lat/0008008.

13. M. Oevers, C. Davies, and J. Shigemitsu, these proceedings, hep-lat/0009031.

14. C. Bernard et al., Phys. Rev. D55 (1997) 6861.

15. J. Engels et al., Phys. Lett. B396 (1997) 210.

16. F. Karsch, E. Laermann, and A. Peikert, Phys. Lett. B478 (2000) 447.

17. CP-PACS Collaboration, in preparation.

18. Y. Iwasaki, Nucl. Phys. B258 (1985) 141;
Univ. of Tsukuba report UTHEP-118 (1983) unpublished.

19. B. Sheikholeslami and R. Wohlert, Nucl. Phys. B259 (1985) 572.

20. S. Aoki, Phys. Rev. D30 (1984) 2653; Phys. Rev. Lett. 57 (1986) 3136; Nucl. Phys. B314 (1989) 79.

21. S. Aoki, A. Ukawa, and T. Umemura, Phys. Rev. Lett. 76 (1996) 873.

22. C. Bernard et al., Phys. Rev. D46 (1992) 4741; C. Bernard et al., Phys. Rev. D49 (1994) 3574; T. Bulm et al., Phys. Rev. D50 (1994) 3377.

23. Y. Iwasaki et al., Phys. Rev. D54 (1996) 7010. 24. CP-PACS Collaboration: A. Ali Khan et al., hep-lat/0008011

25. F. Karsch, Phys. Rev. D49 (1994) 3791; F. Karsch and E. Laermann, ibid. D50 (1994) 6954.

26. S. Aoki et al., Phys. Rev. D57 (1998) 3910.

27. E. Laermann, Nucl. Phys. B (Proc. Suppl.) 60A (1998) 180.

28. Y. Iwasaki et al., Phys. Rev. Lett. 78 (1997) 179.

29. M. Bochicchio et al., Nucl. Phys. B262 (1985) 331.

30. S. Itoh et al., Nucl. Phys. B274 (1986) 33.

31. K. Kanaya and S. Kaya, Phys. Rev. D51 (1995) 2404.

32. D. Toussaint, Phys. Rev. D55 (1997) 362.

33. C. Bernard et al., Phys. Rev. D61 (2000) 054503.

34. J. Kogut, J.-F. Lagaë, and D. K. Sinclair, Phys. Rev. D58 (1998) 034504; J. Kogut and D. K. Sinclair, hep-lat/0005007.

35. J. Engels et al., hep-lat/0006023.

36. F. Karsch, Nucl. Phys. B(Proc.Suppl.) 83-84 (2000) 14.

37. C. Bernard et al., Phys. Rev. D56 (1997) 5584 and references therein.

38. F. Karsch et al., these proceedings, heplat/0010040.

39. R.G. Edwards and U.M. Heller, Phys. Lett. B462 (1999) 132.

40. P.M. Vranas, Nucl. Phys. B(Proc.Suppl.) 83$84(2000) 414$.

41. R.G. Edwards, U.M. Heller, and T.R. Klassen, Nucl. Phys. B517 (1998) 377. 